\title{
Use of clofarabine for acute childhood leukemia
}

\author{
This article was published in the following Dove Press journal: \\ Biologics: Targets \& Therapy \\ 23 June 2010 \\ Number of times this article has been viewed
}

\section{A Pession \\ R Masetti \\ K Kleinschmidt \\ A Martoni \\ Pediatric Oncology and Hematology "Lalla Seràgnoli", University of Bologna, Italy}

\begin{abstract}
A second-generation of purine nucleoside analogs, starting with clofarabine, has been developed in the course of the search for new therapeutic agents for acute childhood leukemia, especially for refractory or relapsed disease. Clofarabine is a hybrid of fludarabine and cladribine, and has shown to have antileukemic activity in acute lymphoblastic leukemia as well as in myeloid disorders. As the only new antileukemic chemotherapeutic agent to enter clinical use in the last 10 years, clofarabine was approved as an orphan drug with the primary indication of use in pediatric patients. Toxicity has been tolerable in a heavily pretreated patient population, and clofarabine has been demonstrated to be safe, both as a single agent and in combination therapies. Liver dysfunction has been the most frequently observed adverse event, but this is generally reversible. Numerous Phase I and II trials have recently been conducted, and are still ongoing in an effort to find the optimal role for clofarabine in various treatment strategies. Concomitant use of clofarabine, cytarabine, and etoposide was confirmed to be safe and effective in two independent trials. Based on the promising results when used as a salvage regimen, clofarabine is now being investigated for its potential to become part of frontline protocols.
\end{abstract}

Keywords: clofarabine, pediatric acute lymphoblastic leukemia, pediatric acute myeloid leukemia

\section{Introduction}

Acute leukemia is the most common pediatric malignancy. ${ }^{1-5}$ Significant improvements in frontline treatment protocols for both childhood acute lymphoblastic leukemia $(\mathrm{ALL})^{2}$ and acute myeloid leukemia (AML) ${ }^{6}$ have led to a marked increase in cure rates. Currently, a long-term remission can be achieved in about $80 \%$ of children affected by $\mathrm{ALL}^{7-11}$ and approximately $50 \%$ of those with AML. ${ }^{12-15}$ However, in cases of primary refractory disease, early systemic relapse, or multiple relapses, salvage regimens have a very dismal outcome. ${ }^{16,17}$ Because relapsed leukemia represents the fourth most common pediatric malignancy, ${ }^{18}$ new therapeutic options for this important group of patients are needed. In this context, clofarabine is the first and only anticancer drug to be approved for use in children before use in adults for more than 10 years. ${ }^{19}$ It is primarily indicated as a single agent for the treatment of pediatric patients aged 1-21 years with relapsed or refractory ALL after at least two prior regimens, and was approved by the US Food and Drug Administration (FDA) in December 2004, and by the European Medicinal Evaluation Agency in May 2006.
Correspondence: R Masetti

Pediatric Oncology and Hematology

"Lalla Seràgnoli", University

of Bologna, Bologna, Italy

$\mathrm{Tel}+390516364016$

$\mathrm{Fax}+39051636449$

Email riccardo.masetti@gmail.com 


\section{Drug development and physicochemical properties}

Clofarabine (2-chloro-2'-fluoro-2'-deoxy-9- $\beta$-D-arabinofuranosyladenine) is a second-generation purine nucleoside analog, thus acting as an antimetabolite. This molecule was developed to overcome the limitations conferred by the toxicity, especially dose-limiting neurotoxicity, known with the structurally related analogs, cladribine and fludarabine, ${ }^{20-22}$ and also to incorporate at the same time the best qualities of both congeners. ${ }^{23}$ Like other purine analogs, clofarabine retains the 2-halogenated aglycone, which renders these compounds resistant to deamination by adenosine deaminase. In addition, clofarabine contains a second halogen atom, fluorine, at the 2'-position of the carbohydrate, which inhibits glycosidic linkage in order to protect it from bacterial purine nucleoside phosphorylase and from acid hydolysis. ${ }^{24,25}$ This chemical configuration prevents release of neurotoxic halogenated adenine, which is the reason for the dose-limiting neurotoxic side effects of similar nucleoside analogs. ${ }^{20}$ The stability of clofarabine in an acidic environment makes for a potential orally available anticancer drug, and there is ongoing research in this area. ${ }^{24}$

\section{Mechanism of action}

To be able to exert its cytotoxic effect, clofarabine requires intracellular phosphorylation by deoxycytidine kinase (dCK) to its active triphosphate form, showing a greater affinity than fludarabine and cladribine for rate-limiting activating $\mathrm{dCK}^{26,27}$ Whereas the congeners of clofarabine primarily inhibit DNA polymerases (fludarabine) or ribonucleotide reductase (cladribine), clofarabine inhibits DNA synthesis and repair by both mechanisms. ${ }^{18,28,29}$ This mechanism leads to depletion of the intracellular deoxynucleotide triphosphate pool, as a result of which clofarabine self-potentiates the incorporation of clofarabine triphosphate into DNA, thereby amplifying the effectiveness of DNA synthesis inhibition. ${ }^{30,31}$ Additionally, this mechanism has an apoptotic effect via release of proapoptotic factors, such as mitochondrial cytochrome $\mathrm{C},{ }^{25,32}$ caused by damage of the integrity of the mitochondrial membrane. . $33,34^{2}$ Induction of the apoptotic pathway leads to programmed cell death, even in nonproliferating human lymphocytes, and could therefore be in part the explanation for the enhanced cytotoxic activity of clofarabine. ${ }^{33,35}$

\section{Pharmacokinetics and drug metabolism}

The highest concentration of clofarabine in plasma occurs at the end of infusion, as known from other nucleoside analogs. ${ }^{36}$ The inhibition rate of DNA synthesis ranged from
$75 \%-95 \%$ (at the end of infusion) when $22.5-55 \mathrm{mg} / \mathrm{m}^{2}$ doses have been administered. ${ }^{31}$ When lower doses (22.5 and $30 \mathrm{mg} / \mathrm{m}^{2}$ ) were administered, a partial recovery of inhibition of DNA synthesis was observed after 24 hours, whereas at higher dose levels (40 and $55 \mathrm{mg} / \mathrm{m}^{2}$ ) inhibition was maintained, demonstrating a dose-dependent effect on degree and maintenance of DNA synthesis inhibition during therapy with clofarabine. ${ }^{37}$ Patient-dependent parameters shown to influence the pharmacokinetic profile of clofarabine are body weight and white blood cell (WBC) count. While the observed increase in clofarabine plasma concentration in cases of a WBC decrease does not seem to be significant enough to justify modification of the treatment regimen as a function of WBC count, administration of the dose on a per square meter or per body weight basis is crucial. ${ }^{38}$ Clofarabine is $47 \%$ bound to plasma protein, of which $27 \%$ is serum albumin, resulting in wide tissue distribution. ${ }^{39}$ Once intracellular, accumulation of clofarabine triphosphate, the active form of clofarabine, in the blasts of patients with refractory leukemia has been demonstrated in several studies. ${ }^{37,38}$ Because the highest concentrations of dCK are found in lymphoid tissues, a sustained antileukemic activity would be expected,,$^{38}$ enhanced by the prolonged intracellular half-life of 24 hours for clofarabine triphosphate, in contrast with fludarabine and cladribine, which have median half-lives of only $8-10$ hours. ${ }^{18,37}$ Based on 24-hour urine collection, $49 \%-60 \%$ of the dose is excreted in the urine unchanged. Metabolism via the cytochrome P450 system has not been demonstrated, so the pathways for nonrenal excretion of clofarabine remain unknown for the moment. ${ }^{37}$ The terminal half-life of clofarabine is estimated to be 5.2 hours. ${ }^{18}$

\section{Clinical efficacy Preclinical studies}

Initial interest in performing clinical efficacy and safety studies for clofarabine was rather subdued, as a result of the unfavorable toxicity-efficacy ratio observed for fludarabine and cladribine. The antileukemic activity of fludarabine has been established, but the toxic side effects at therapeutic dosage were not acceptable (death of $4 / 9$ patients due to severe neurologic side effects in one study). ${ }^{40}$ Because clofarabine has been shown to be even more lipophilic than cladribine, ${ }^{41}$ it has been presumed that its neurotoxicity would be at least as severe as that for the other nucleoside analogs, attributing at most a role for the treatment of lymphoproliferative disorders in the central nervous system. ${ }^{31}$ Surprisingly, the accumulation of clofarabine in brain tissue of mice was found to be lower than for cladribine. ${ }^{42}$ The few differences in the chemical structure were decisive. 
The early intention of Kantarjian et al in 1992 to conduct a Phase I study in humans, ${ }^{23}$ after the synthesis of clofarabine earlier that year, ${ }^{43}$ was delayed when the FDA requested that animal toxicology studies in two animal models (mice and dogs) be done first. These studies identified a safe starting dose of $15 \mathrm{mg} / \mathrm{m}^{2}$ daily for five days (1/10th of $\mathrm{LD}_{10}$ in mice) for human Phase I clinical trials (Kantarjian, unpublished data), ${ }^{23}$ approved by the FDA in $1998 .{ }^{23}$

\section{Early clinical studies}

The first Phase I study of clofarabine was performed in 51 adult patients with refractory/relapsed solid or hematologic malignancies to define the dose-limiting toxicity (DLT) of the drug and to determine the maximum tolerated dose (MTD) for acute and chronic leukemias, myeloma, and solid tumors. ${ }^{44}$ The MTD for patients with solid tumors was determined to be $2 \mathrm{mg} / \mathrm{m}^{2}$, showing that the starting dose of $15 \mathrm{mg} / \mathrm{m}^{2}$ was too high, with the DLT being myelosuppression. However, the overestimation of the safe starting dose is known to be due to the differing pharmacokinetic profiles for the drug in dogs and humans. ${ }^{45,46}$ On the other hand, the MTD for acute leukemia was determined at $40 \mathrm{mg} / \mathrm{m}^{2} /$ day, with the DLT being hepatotoxicity. ${ }^{44}$ This marked difference of a 20 -fold higher MTD in patients with leukemia versus those with solid tumors was unattended and exceeded the usual difference of a two- to seven-fold higher MTD for other agents. Because transient myelosuppression and cytopenia during the treatment of leukemia are unavoidable, these are not considered to be conventional dose-limiting side effects. Thirteen of 32 patients with leukemia in this Phase I study had ALL. Their overall response rate was $15 \%$, consisting of one complete remission (CR) and one $\mathrm{CR}$ without platelet recovery (CRp).

Based on these results, a parallel Phase I trial for pediatric patients with advanced leukemia was opened, including 25 patients affected by relapsed or refractory leukemia (17 ALL, eight AML). ${ }^{47}$ Six dose levels $\left(11.25 \mathrm{mg} / \mathrm{m}^{2}\right.$, $15 \mathrm{mg} / \mathrm{m}^{2}, 30 \mathrm{mg} / \mathrm{m}^{2}, 40 \mathrm{mg} / \mathrm{m}^{2}, 52 \mathrm{mg} / \mathrm{m}^{2}$, and $70 \mathrm{mg} / \mathrm{m}^{2}$ ) were investigated to define the DLT and MTD for children. The MTD was established to be $52 \mathrm{mg} / \mathrm{m}^{2} /$ day for five days, and the DLT was identified to be reversible Grade 3 hepatotoxicity and skin rash. Hematopoietic stem cell transplantation (HSCT) either before $(n=9)$ or after $(n=7)$ clofarabine did not cause increased toxicity. The majority of the patient cohort (20/25) received more than one cycle (12 patients had two cycles, seven had three cycles, and two patients had eight cycles), and this was not associated with cumulative hepatotoxicity. The overall response (OR) rate was $32 \%$, with five patients achieving a $\mathrm{CR}(\mathrm{ALL}, \mathrm{n}=4$; AML, $\mathrm{n}=1)$ and three having a partial response $(\mathrm{PR}, \mathrm{ALL}, \mathrm{n}=1 ; \mathrm{AML}, \mathrm{n}=2)$.

Two open-label, multicenter, nonrandomized Phase II trials were opened in 2002, aiming to establish the efficacy and safety profile of clofarabine in pediatric patients (age 1-21 years at diagnosis), affected by ALL (median age 12 years) or AML (median age 13 years), and with relapsed disease or disease refractory to standard therapies. ${ }^{19,48}$

The study of the efficacy of clofarabine in ALL enrolled 61 patients who had previously received a median of three (range 2-6) treatment regimens, and nearly a third of these patients had received at least one prior HSCT regimen (30\%). Immunophenotyping showed that $79 \%$ of patients were B precursor/B cell lineage, $8 \%$ were $\mathrm{T}$ cell lineage, and $13 \%$ had acute leukemia with other/unknown cell lineage. ${ }^{19}$ All patients received intravenous (IV) clofarabine $52 \mathrm{mg} / \mathrm{m}^{2} /$ day as a single agent on five consecutive days for up to 11 cycles (median two). The median time between two cycles was 28 (range 12-55) days. The results of this trial showed an OR rate of 20\% (seven CR and five CRp), and an additional $\mathrm{PR}$ rate of $10 \%$. CR patients who proceeded to HSCT maintained their CR for a median period of seven months. The median CR duration was only two months when treatment with clofarabine was not followed by HSCT. Nine patients proceeded to transplantation after clofarabine therapy. The most frequently observed Grade $\geq 3$ adverse events were febrile neutropenia (49\%), followed by hypokalemia $(46 \%)$, elevated aspartate transaminase $(38 \%)$ or alanine transaminase (43\%), anorexia (20\%), hypotension (18\%), nausea $(16 \%)$, hyperbilirubinemia $(16 \%)$, and neutropenia $(15 \%)$. Systemic inflammatory response-like or cytokine release-like events (including respiratory distress, fever, and hypotension), skin rash, and hand-food syndrome were also observed. ${ }^{19}$

The results of this study were the basis for the accelerated approval of clofarabine in the US and in Europe for the treatment of pediatric ALL in at least its second relapse. Similar trials were initiated and broadly confirmed the existing data, eg, in the similarly designed European Phase II nonrandomized, open-label study, in which 96 courses of clofarabine at the same dosage of $52 \mathrm{mg} / \mathrm{m}^{2} /$ day were administered to 53 pediatric patients with relapsed/refractory ALL. ${ }^{49}$ In that study, an OR rate of $26 \%$ was achieved (six CR, seven CRp, and one PR). Four patients (one CR and three CRp) proceeded to HSCT. The most frequent adverse events were febrile neutropenia (50\%), seizures (4\%), and sepsis and hepatotoxicity each in 3\%. The lower number of hepatic adverse events in comparison with the US trial may have 
been due to a more heavily pretreated patient population in the US study (median three versus two prior regimens). ${ }^{49}$

Results for the AML arm in the study by Jeha et al have recently been published, describing a treatment regimen conducted in 42 patients with refractory or relapsed AML, where clofarabine was used as a single agent at $52 \mathrm{mg} / \mathrm{m}^{2} /$ day for five consecutive days. ${ }^{48}$ In this study, the OR rate was $26 \%$, with one CRp and 10 PR. Six of the responders were patients who had been refractory to the preceding therapy. Thirteen patients, of whom seven were responders, proceeded to HSCT. The median response duration was 20 weeks, whereas the survival duration of transplanted patients ranged from 24 to $\geq 160$ weeks. The overall toxicity profile was comparable with that observed in other studies (Table 1), but five patients died as a result of an adverse event. ${ }^{48}$

\section{Postmarketing combination studies}

In view of the encouraging results of the trials with clofarabine as a single agent, and with regard to its mechanism of action, several studies investigated the possibility of incorporating clofarabine into rational combinations with conventional anticancer drugs. ${ }^{50,51}$

\section{Clofarabine combined with alkylating agents}

An increased cytotoxic effect could be expected using a combination of clofarabine and alkylating agents, such as cyclophosphamide. Repair of the DNA damage caused by the alkylating agents would be impeded due to the ability of clofarabine to inhibit DNA synthesis/repair. A synergistic effect was demonstrated first in in vitro studies, ${ }^{28}$ and was successively confirmed in clinical trials. A Phase I dose-escalating pilot study of clofarabine used in combination with cyclophosphamide and etoposide was conducted by Hijiya et al to determine the MTD and DLT for this new treatment regimen in pediatric patients with refractory or relapsed acute leukemia. ${ }^{52}$ The preliminary data for this Phase I study were reported while a Phase II study of the same treatment regimen was already ongoing. ${ }^{52}$ In the Phase I trial, 25 patients (20 with ALL and five with AML) were enrolled, and five dosing cohorts were evaluated using escalating doses of clofarabine $20-40 \mathrm{mg} / \mathrm{m}^{2} /$ day, etoposide $75-100 \mathrm{mg} / \mathrm{m}^{2} /$ day, and cyclophosphamide $340-440 \mathrm{mg} / \mathrm{m}^{2} /$ day. ${ }^{52}$ An OR rate of $64 \%$ was achieved (ALL 55\%, AML 100\%), consisting of a CR in 10 patients (nine with ALL, one with AML) and a CRp in six patients (two with ALL 2, four with AML). Of these 16 patients, nine proceeded to HSCT; the median duration of remission for these patients was 18.2 weeks. The response rate was $69 \%$ in patients with B cell precursor ALL, documented in $20 \%$ of patients with T cell ALL. A MTD was not reached, and the recommended Phase II doses were clofarabine $40 \mathrm{mg} / \mathrm{m}^{2} /$ day, cyclophosphamide $440 \mathrm{mg} / \mathrm{m}^{2} /$ day, and etoposide $100 \mathrm{mg} / \mathrm{m}^{2} /$ day. The most common adverse events (all grades) were nausea (80\%), vomiting (72\%), febrile neutropenia $(64 \%)$, pyrexia (48\%), diarrhea (44\%), skin rash (40\%), and transaminase elevation (38\%). Because of unexpected hepatotoxicity (three cases of veno-occlusive disease and one of hyperbilirubinemia), the Phase II portion of the study was amended to exclude patients with, eg, viral hepatitis, liver cirrhosis, hyperbilirubinemia, and prior HSCT. $^{52}$ This trial is evaluating the safety and efficacy of this combination regimen in ALL patients, and is ongoing.

Table I Patients with Grade $\geq 3$ adverse events in all cycles

\begin{tabular}{|c|c|c|c|c|c|}
\hline & \multicolumn{5}{|c|}{ Reference study } \\
\hline & Phase $I^{47}$ & $\begin{array}{l}\text { Phase II } \\
\text { ALL'19 }\end{array}$ & $\begin{array}{l}\text { Phase II } \\
\text { AML }^{48}\end{array}$ & $\begin{array}{l}\text { CLO-CPM- } \\
\text { VP- } 16^{52}\end{array}$ & $\begin{array}{l}\text { CLO-CPM- } \\
\text { VP- } 16^{53}\end{array}$ \\
\hline \multicolumn{6}{|l|}{ Adverse event } \\
\hline Hypertransaminasemia & $28 \%$ & $43 \%$ & $43 \%$ & $38 \%$ & $24 \%$ \\
\hline Hyperbilirubinemia & & $16 \%$ & $12 \%$ & $0 \%$ & \\
\hline Febrile neutropenia & n.i. & $49 \%$ & $55 \%$ & $64 \%$ & n.i. \\
\hline Infections & n.i. & $69 \%$ & $67 \%$ & $72 \%$ & $32 \%$ \\
\hline Nausea/vomiting & $16 \%$ & $16 \%$ & $17 \%$ & $12 \%$ & n.i. \\
\hline Hypokalemia & n.i. & $46 \%$ & $40 \%$ & $24 \%$ & n.i. \\
\hline Pericardial effusion & n.i. & $29 \%$ & $39 \%$ & n.i. & $0 \%$ \\
\hline Decrease in left ventricular systolic function & n.i. & $17 \%$ & $32 \%$ & n.i. & $0 \%$ \\
\hline Skin rash & $4 \%$ & $10 \%$ & n.i. & $0 \%$ & $0 \%$ \\
\hline Hypotension & n.i. & $18 \%$ & $17 \%$ & n.i. & n.i. \\
\hline Hypertension & n.i. & $10 \%$ & n.i. & n.i. & n.i. \\
\hline AE-related death $(n)$ & $0 \%$ & $8 \%(5)$ & $12 \%(5)$ & $12 \%(3)$ & $0 \%$ \\
\hline
\end{tabular}

Abbreviations: AE, adverse events; CLO, clofarabine; CPM, cyclophosphamide; VP-I6, etoposide; n.i., not indicated. 
The second open-label, multicenter trial investigating the possible complementary effect of a clofarabine-cyclophosp hamide-etoposide combination was a nonrandomized Phase II efficacy and safety study performed by Locatelli et al. ${ }^{53}$ This study included 25 pediatric patients (median age 12.5 years $)$ with either refractory $(\mathrm{n}=17)$ or multiple relapsed $(\mathrm{n}=8)$ ALL. The treatment regimen, administered on five consecutive days, comprised a two-hour IV infusion of clofarabine $40 \mathrm{mg} / \mathrm{m}^{2} /$ day, followed by a onehour infusion of cyclophosphamide $400 \mathrm{mg} / \mathrm{m}^{2} /$ day and a two-hour infusion of etoposide $150 \mathrm{mg} / \mathrm{m}^{2} /$ day. In this trial, an OR rate of $56 \%$ was achieved, consisting of 13 patients (52\%) achieving a CR and another achieving a CRp (4\%). For more than half of the CR patients (53\%), the remission duration was sufficient to allow them to proceed to HSCT. As in the study by Hijiya et al the probability of reaching a CR/CRp was significantly higher in patients with $\mathrm{B}$ cell precursor ALL (76\%) than in those with $\mathrm{T}$ lineage ALL $(12 \%)$. The most frequent adverse events were febrile neutropenia, reversible liver toxicity, and mucositis. No case of veno-occlusive disease, tumor lysis syndrome, or cytokinerelease syndrome was reported. This favorable tolerability may be due to the careful prophylaxis regimen used in this trial, including prophylactic steroid administration to prevent cytokine-release syndrome when $>30 \times 10^{9}$ blast cells $/ \mathrm{L}$ in peripheral blood was present at the time of treatment.

\section{Clofarabine combined with other nucleoside analogues}

Playing on the concept that clofarabine effectively inhibits the ribonucleotide reductase, an association with the pyrimidine analog, cytarabine, was performed.$^{54}$ In in vitro experiments, it has been observed that pretreatment with clofarabine leads to enhanced intracellular cytarabine triphosphate levels via indirect upregulation of $\mathrm{dCK}$, caused by a decreased dNTP pool (ribonucleotide reductase inhibition).$^{54}$ Phase I-II studies of clofarabine in combination with cytarabine in an adult patient population with relapsed or refractory acute leukemia have been performed. ${ }^{55,56}$ Clofarabine $40 \mathrm{mg} / \mathrm{m}^{2} /$ day was given for five days as a one-hour IV infusion, followed four hours later by cytarabine $1 \mathrm{~g} / \mathrm{m}^{2} /$ day as a two-hour infusion for five days. This treatment regimen was shown to be safe and effective in 32 patients with relapsed leukemia (two with ALL and 25 with AML, four with high-risk myelodysplastic syndrome, and one with blast-phase chronic myeloid leukemia). ${ }^{55}$ The same investigators have also investigated a salvage regimen of idarubicin-clofarabine \pm cytarabine for adult patients with AML. ${ }^{57}$ A single case report of clofarabine-cytarabine in a nine-year-old boy with multiple relapsed T cell ALL showed a complete morphologic and cytogenetic remission using one cycle of this combination. ${ }^{58}$

A recently closed pediatric Phase I-II study has investigated the safety of clofarabine-cytarabine in pediatric patients with AML in first relapse and ALL in at least second relapse. ${ }^{59}$ In this trial, a fixed cytarabine dose of $1 \mathrm{~g} / \mathrm{m}^{2}$ was administered from days $1-5$ of the cycle, whereas clofarabine was administered on days 2-6 at varying doses. As in the adult study, clofarabine was given four hours before the infusion of cytarabine, taking advantage of the synergistic mechanism of action of these agents. The first cohort included 10 patients (eight with ALL and two with AML), and the starting dose of clofarabine was the adult singleagent MTD of $40 \mathrm{mg} / \mathrm{m}^{2}$. This dosage could be escalated to $52 \mathrm{mg} / \mathrm{m}^{2}$, which was then administered to the second patient cohort (three with ALL and seven with AML). The doses used at the end of the Phase I trial have been defined to be the MTD for this drug combination. The observed toxicity profile was comparable with that previously described. It was concluded that the clofarabine-cytarabine combination is safe and tolerable. ${ }^{59}$

Started in 2008, an ongoing Phase II randomized trial of clofarabine-cytarabine versus conventional induction therapy (cytarabine-daunorubicin-etoposide) in patients with newly diagnosed AML is investigating a role for clofarabine in the early phase of antileukemic treatment. A more favorable toxicity profile could be expected using this combination in a patient population not having undergone chemotherapy previously, and hopefully with a better final outcome.

\section{Clofarabine-anthracycline combination therapy}

The ability of clofarabine to inhibit DNA strand elongation makes it suitable for use in combination with anthracyclines, thereby taking advantage of the anthracycline's ability to induce DNA intercalation and strand breaks. Early data for a possible synergistic effect have been reported from an in vitro study using oxaliplatin-fludarabine. ${ }^{60}$ A Phase I/II study of clofarabine in combination with cytarabine and liposomal daunorubicin given to children affected by relapsed/refractory AML is ongoing.

\section{Clofarabine as a conditioning regimen for HSCT}

In an attempt to enhance the cytotoxic effect of clofarabine whilst reducing treatment-related toxicity, clofarabine is also being investigated as part of conditioning regimens. ${ }^{61} \mathrm{~A}$ Phase I 
trial at the St Jude Children's Research Hospital is presently determining the MTD and DLT of clofarabine when used in combination with melphalan and thiotepa in a pretransplant conditioning regimen. Several studies integrating clofarabine into conditioning regimens are also ongoing in adults.

\section{Toxicity profile of clofarabine}

On the basis of the studies reported to date, the most common Grade $\geq 3$ adverse events with clofarabine are hepatotoxicity (elevated transaminases and/or hyperbilirubinemia), febrile neutropenia, and infections. A detailed description of the adverse event distribution in the main studies of clofarabine in pediatric patients is outlined in the Table 1 .

Hepatotoxicity was the DLT for clofarabine in the Phase I trial, ${ }^{47}$ and was observed in all Phase II studies, both in singleuse and in combination regimens. ${ }^{19,48,52,53}$ Hepatic dysfunction was usually reversible, showing a peak around days 5-7, and recovery by days $15-16$ (< Grade 2$){ }^{18,19}$ In some cases, however, severe and potentially life-threatening hepatotoxicity occurred with the use of clofarabine. In the Phase II study, performed on the basis of the Phase I combination study of clofarabine-cyclophosphamide-etoposide by Hijiya et al three of the first eight patients enrolled developed veno-occlusive disease, and there was one case of hyperbilirubinemia. Thereafter, the Phase II portion of the trial was amended as described above and so far, none of the further seven patients enrolled have experienced severe hepatotoxicity. ${ }^{52}$ As a result of the studies performed until now, close monitoring of liver function is recommended during and after therapy with clofarabine, and the potentially cumulative hepatotoxicity should be considered when using other drugs known to compromise liver function.

In the Phase II study by Jeha et al several cases of systemic inflammatory response or cytokine release-like events, capillary leak syndrome, and severe tumor lysis syndrome, including a fatal outcome, have been associated with the use of clofarabine. ${ }^{19}$ To prevent this type of adverse event, Locatelli et al performed a study in which steroids have been administered in a prophylactic manner when $>30 \times 10^{9}$ blasts/L were counted in peripheral blood at the time of treatment. None of the enrolled patients experienced tumor lysis, cytokine-release, or systemic inflammatory syndrome..$^{53}$

In addition, to prevent infection-related complications, a broad-spectrum antibiotic, antiviral, and antifungal prophylactic regimen should be instituted in all patients treated with clofarabine.

\section{Resistance to clofarabine}

Although the efficacy of clofarabine is not restricted to a specific leukemia subtype, and CRs have been achieved in both AML and ALL patients, some differences in response pattern have been seen. It is conspicuous that the OR rate in the Hijiya's study group was $69 \%$ in patients with B cell precursor ALL $(\mathrm{CR}=9)$, and only $20 \%$ in five patients with the $\mathrm{T}$ lineage subtype $(\mathrm{CRp}=1) .{ }^{52}$ An even larger gap in terms of response was reported by Locatelli et al who reported that a CR/CRp was achieved by $76 \%$ of children with B cell precursor ALL, whereas only $12 \%$ of those with $\mathrm{T}$ cell leukemia responded to therapy with clofarabine. ${ }^{53}$ These data could be indicative of a possible resistance mechanism inherent in $\mathrm{T}$ lineage leukemic blasts. An observation supporting such a hypothesis is the conclusion of an in vitro study, where clofarabine appeared to be slightly more effective in B lymphoblasts than in T blasts. ${ }^{62}$ Furthermore, pharmacologic studies have reported cross-resistance of clofarabine-resistant cell lines to other antimetabolites, such as cytarabine or fludarabine, which are phosphorylated by dCK. ${ }^{32}$ Reduced dCK activity has been shown to be directly correlated with resistance to clofarabine, because those cell lines did not consistently form detectable levels of triphosphates. ${ }^{31} \mathrm{dCK}$ deficiency has been demonstrated at the level of enzymatic activity, at the protein level, and also at the mRNA expression level for resistant cell lines. ${ }^{31}$ However, the hypothesis that DNA methylation might be responsible for the decreased dCK level could not be confirmed, and no mutations in the coding region of the $\mathrm{dCK}$ have been detected so far. ${ }^{32}$ Further studies will be necessary to explain resistance mechanisms to clofarabine, and to indicate consequently which leukemic form is sensitive to therapy with clofarabine and for which ones alternative treatment strategies are needed.

\section{Summary and future directions}

Clofarabine is a recent second-generation purine adenosine analog with important therapeutic efficacy not restricted to a specific leukemic subtype, and with responses obtained even in patients with prior refractory disease. It has been shown to be safe when used as a single agent and in rational combinations with conventional chemotherapeutic drugs. The toxicity profile is tolerable in a heavily pretreated patient population. A role for clofarabine in frontline protocols is presently being investigated. Other studies are exploring the potential of clofarabine to become a component of conditioning regimens for hematopoietic stem cell transplantation. Defining the optimal time point and the most appropriate 
treatment regimen for the use of clofarabine will be the subject of future studies.

\section{Disclosure}

The authors report no conflicts of interest in this work.

\section{References}

1. Pui CH. Childhood leukemias. N Engl J Med. 1995;332(24):1618-1630.

2. Pui CH, Evans WE. Treatment of acute lymphoblastic leukemia. $N$ Engl J Med. 2006;354(2):166-178.

3. Kersey JH. Fifty years of studies of the biology and therapy of childhood leukemia. Blood. 1997;90(11):4243-4251.

4. McNeil DE, Cote TR, Clegg L, Mauer A. SEER update of incidence and trends in pediatric malignancies: Acute lymphoblastic leukemia. Med Pediatr Oncol. 2002;39(6):554-557.

5. Ries LAG, Melbert D, Krapcho M, et al. SEER Cancer Statistics Review, 1975-2005, National Cancer Institute: Bethesda, MD; Updated 2008. Available from: http://seer.cancer.gov/csr/1975_2005/ Accessed on May 30, 2010.

6. Kaspers GJ, Creutzig U. Pediatric acute myeloid leukemia: International progress and future directions. Leukemia. 2005;19:2025-2029.

7. Pui CH, Pei D, Sandlund JD, et al. Long-term results of St Jude Total Therapy Studies 11, 12, 13A, 13B, and 14 for childhood acute lymphoblastic leukaemia. Leukemia. 2010;24(2):371-382.

8. Schrappe M, Reiter A, Zimmermann M, et al. Long-term results of four consecutive trials in childhood ALL performed by the ALL-BFM study group from 1981 to 1995. Berlin-Frankfurt-Münster. Leukemia. 2000;14(12):2205-2222.

9. Schultz KR, Pullen DJ, Sather HN, et al. Risk- and response-based classification of childhood B-precursor acute lymphoblastic leukemia: A combined analysis of prognostic markers from the Pediatric Oncology Group (POG) and Children's Cancer Group (CCG). Blood. 2007; 109(3):926-935.

10. Pession A, Valsecchi MG, Masera G, et al. Long-term results of a randomized trial on extended use of high dose L-asparaginase for standard risk childhood acute lymphoblastic leukemia. J Clin Oncol. 2005; 23(28):7161-7167.

11. Aricò M, Valsecchi MG, Conter V, et al. Improved outcome in high-risk childhood acute lymphoblastic leukemia defined by prednisone-poor response treated with double Berlin-Frankfurt-Muenster protocol II. Blood. 2002;100(2):420-426.

12. Pession A, Rondelli R, Basso G, et al. Treatment and long-term results in children with acute myeloid leukaemia treated according to the AIEOP AML protocols. Leukemia. 2005;19(12):2043-2053.

13. Creutzig U, Zimmermann M, Ritter J, et al. Treatment strategies and long-term results in paediatric patients treated in four consecutive AML-BFM trials. Leukemia. 2005;19(12):2030-2042.

14. Gibson BE, Wheatley K, Hann IM, et al. Treatment strategy and longterm results in paediatric patients treated in consecutive UK AML trials. Leukemia. 2005;19(12):2130-2138.

15. Ravindranath Y, Chang M, Steuber CP, et al. Pediatric Oncology Group (POG) studies of acute myeloid leukemia (AML): A review of four consecutive childhood AML trials conducted between 1981 and 2000. Leukemia. 2005;19(12):2101-2116.

16. Ko RH, Ji L, Barnette P, et al. Outcome of patients treated for relapsed or refractory acute lymphoblastic leukemia: A Therapeutic Advances in Childhood Leukemia Consortium Study. J Clin Oncol. 2010;28(4):648-654.

17. Zwaan CM, Reinhardt D, Zimmerman M, et al. Salvage treatment for children with refractory first or second relapse of acute myeloid leukaemia with gemtuzumab ozogamicin: Results of a phase II study. Br J Haematol. 2010;148(5):768-776.

18. Jeha S, Kantarjian H. Clofarabine for the treatment of acute lymphoblastic leukemia. Expert Rev Anticancer Ther. 2007;7(2):113-118.
19. Jeha S, Gaynon PS, Razzouk BI, et al. Phase II study of clofarabine in pediatric patients with refractory or relapsed acute lymphoblastic leukemia. J Clin Oncol. 2006;24(12):1917-1923.

20. Cheson BD, Vena DA, Foss FM, Sorensen JM. Neurotoxicity of purine analogs: A review. J Clin Oncol. 1994;12(10):2216-2228.

21. Vahdat L, Wong ET, Wile MJ, Rosenblum M, Foley KM, Warrell RP Jr. Therapeutic and neurotoxic effects of 2-chlorodeoxyadenosine in adults with acute myeloid leukemia. Blood. 1994;84(10): 3429-3434.

22. Warrell RP Jr, Berman E. Phase I and II study of fludarabine phosphate in leukemia: Therapeutic efficacy with delayed central nervous system toxicity. J Clin Oncol. 1986;4(1):74-79.

23. Kantarjian HM, Jeha S, Gandhi V, Wess M, Faderl S. Clofarabine: Past, present, and future. Leuk Lymphoma. 2007;48(10):1922-1930.

24. Carson DA, Wasson DB, Esparza LM, et al. Oral antilymphocyte activity and induction of apoptosis by 2-chloro-2'-arabino-fluoro-2'deoxyadenosine. Proc Natl Acad Sci U S A. 1992;89(7):2970-2974.

25. Parker WB, Shaddix SC, Chang CH, et al. Effects of 2-chloro-9-(2-deoxy2-fluoro- $\beta$-D-arabinofuranosyl)adenine on K562 cellular metabolism and the inhibition of human ribonucleotide reductase and DNA polymerases by its 5'-triphosphate. Cancer Res. 1991;51(9):2386-2394.

26. Xie KC, Plunkett W. Deoxynucleotide pool deletion and sustained inhibition of ribonucleotide reductase and DNA synthesis after treatment of human lymphoblastoid cells with 2-chloro-9-(2-deoxy-2-fluoro- $\beta$-Darabinofuranosyl)adenine. Cancer Res. 1996;56(13):3030-3037.

27. Parker WB, Shaddix SC, Rose LM, et al. Comparison of the mechanism of cytotoxicity of 2-chloro-9-(2-deoxy-2-fluoro- $\beta$-D-arabinofuranosyl) adenine, 2-chloro-9-(2-deoxy-2-fluoro- $\beta$-D-ribofuranosyl)adenine, and 2-chloro-9-(2-deoxy-2,2-difluoro- $\beta$-D-ribofuranosyl)adenine in CEM cells. Mol Pharmacol. 1999;55(3):515-520.

28. Yamauchi T, Nowak B, Keating M, Plunkett W. DNA repair initiated in chronic lymphocytic leukemia lymphocytes by 4-hydroperoxycyclophosphamide is inhibited by fludarabine and clofarabine. Clin Cancer Res. 2001;7(11):3580-3589.

29. Lech-Maranda E, Korycka A, Robak T. Clofarabine as a novel nucleoside analogue approved to treat patients with haematological malignancies: Mechanism of action and clinical activity. Mini Rev Med Chem. 2009;9(7):805-812.

30. Chang $\mathrm{CH}$, Cheng YC. Effects of deoxyadenosine triphosphate and 9-beta-D-arabinofuranosyl-adenine 5'-triphosphate on human nucleotide reductase from Molt- $4 \mathrm{~F}$ cells and the concept of "self-potentation". Cancer Res. 1980;40(10):3555-3558.

31. ZhenchukA, Lotfi K, Juliusson G, Albertioni F. Mechanisms of anti-cancer action and pharmacology of clofarabine. Biochem Pharmacol. 2009; 78(11):1351-1359.

32. Mansson E, Flordal E, Liliemark J, et al. Down-regulation of deoxycytidine kinase in human leukemic cell lines resistant to cladribine and clofarabine and increased ribonucleotide reductase activity contributes to fludarabine resistance. Biochem Pharmacol. 2003;65(2):237-247.

33. Genini D, Adachi S, Chao Q, et al. Deoxyadenosine analogs induce programmed cell death in chronic lymphocytic leukemia cells by damaging the DNA and by directly affecting the mitochondria. Blood. 2000;96(10):3537-3543.

34. Takahashi T, Shimizu M, Akinaga S. Mechanisms of the apoptotic activity of Cl-F-araA in a human T-ALL cell line, CCRF-CEM. Cancer Chemother Pharmacol. 2002;50(3):193-201.

35. Xie C, Plunkett W. Metabolism and actions of 2-chloro-9-(2-deoxy2-fluoro-beta-D- arabinofuranosyl)-adenine in human lymphoblastoid cells. Cancer Res. 1995;55(13):2847-2852.

36. Plunkett W, Ghandi V. Purine and pyrimidine nucleoside analogs. In: Giacconi G, Schilsky R, Sondel P, editors. Cancer Chemotherapy and Biological Response Modifiers Annual 19. Oxford: Elsevier Science; 2001.

37. Gandhi V, Kantarjian H, Faderl S, et al. Pharmacokinetics and pharmacodynamics of plasma clofarabine and cellular clofarabine triphosphate in patients with acute leukemias. Clin Cancer Res. 2003; 9(17):6335-6342. 
38. Bonate PL, Craig A, Gaynon P, et al. Population pharmacokinetics of clofarabine, a second-generation nucleoside analog, in pediatric patients with acute leukemia. J Clin Pharmacol. 2004;44(11):1309-1322.

39. Reichelová V, Juliusson G, Spasokoukotskaja T, et al. Interspecies differences in the kinetic properties of deoxycytidine kinase elucidate the poor utility of a phase I pharmacologically directed dose-escalation concept for 2-chloro-2'-deoxyadenosine. Cancer Chemother Pharmacol. 1999;36(6):524-529.

40. Warrell R, Berman E. Phase I and II study of fludarabine phosphate in leukemia: Therapeutic efficacy with delayed CNS toxicity. J Clin Oncol. 1986;4(1):74-79.

41. Albertioni F, Hassan M, Silberring J, Liliemark J. Kinetics and metabolism of 2-chloro-2'-deoxyadenosine and 2-chloro-2'-arabino-fluoro-2'deoxyadenosine in the isolated perfused rat liver. Eur J Drug Metab Pharmacokinet. 1995;20(3):225-232.

42. Lindemalm S, Liliemark J, Larsson BS, Albertioni F. Distribution of 2-chloro-2'-deoxyadenosine, 2-chloro-2'-arabino-fluoro-2'deoxyadenosine, fludarabine and cytarabine in mice: A whole-body autoradiography study. Med Oncol. 1999;16(4):239-244.

43. Montgomery JA, Shortnacy-Fowler AT, Clayton SD, et al. Synthesis and biologic activity of 2'-fluoro-2-halo derivates of 9-beta-Darabinofuranosyladenine. J Med Chem. 1992;35(2):397-401.

44. Kantarjian HM, Gandhi V, Kozuch P, et al. Phase I clinical and pharmacology study of clofarabine in patients with solid and hematological cancers. J Clin Oncol. 2003;21(6):1167-1173.

45. Noker P, Duncan G, El Dareer S, et al. Disposition of 9-beta-Darabinofuranosyl-2-fluoradenine 5'-phosphate in mice and dogs. Cancer Treat Rep. 1983;67(5):445-456.

46. Malspeis L, Grever M, Staubus A, et al. Pharmacokinetics of 2-F-ara-A (9-beta-D-arabinofuranosyl-2-fluoroadenine) in cancer patients during the phase I clinical investigation of fludarabine phosphate. Semin Oncol. 1990;17(5):18-32.

47. Jeha S, Gandhi V, Chan KW, et al. Clofarabine, a novel nucleoside analog, is active in pediatric patients with advanced leukemia. Blood. 2004;103(3):784-789.

48. Jeha S, Razzouk B, Rytting M, et al. Phase II study of clofarabine in pediatric patients with refractory or relapsed acute myeloid leukemia. J Clin Oncol. 2009;27(26):4392-4397.

49. Kearns P, Michel G, Nelken B, et al. BIOV-111 a European Phase II trial of clofarabine $\left(\right.$ Evoltra $\left.^{\circledR}\right)$ in refractory and relapsed childhood acute lymphoblastic leukemia. Blood. 2006;108(11): Abstr 1859.
50. Musto P, Ferrara F. Clofarabine: In search of combinations for the treatment of patients with high-risk acute myeloid leukemia. Cancer. 2008; 113(8):1995-1998.

51. Jeha S. Recent progress in the treatment of acute lymphoblastic leukemia: Clofarabine. Hematol Oncol Clin North Am. 2009;23(5):1137-1144.

52. Hijiya N, Gaynon PS, Barry E, et al. A multi-center phase I study of clofarabine, etoposide and cyclophosphamide in combination in pediatric patients with refractory or relapsed acute leukemia. Leukemia. 2009;23(12):2259-2264.

53. Locatelli F, Testi AM, Bernardo E, et al. Clofarabine, cyclophosphamide and etoposide as single-course re-induction therapy for children with refractory/multiple relapsed acute leukaemia. $\mathrm{Br} J$ Haematol. 2009; 147(3):371-378.

54. Cooper T, Ayres M, Nowak B, Gandhi V. Biochemical modulation of cytarabine triphosphate by clofarabine. Cancer Chemother Pharmacol. 2005;55(4):361-368.

55. Faderl S, Gandhi V, O'Brien S, et al. Results of a phase 1-2 study of clofarabine in combination with cytarabine (ara-C) in relapsed and refractory acute leukemias. Blood. 2005;105(3):940-947.

56. Faderl S, Verstovsek S, Cortes J, et al. Clofarabine and cytarabine combination as induction therapy for acute myeloid leukemia (AML) in patients 50 years of age or older. Blood. 2006;108(1):45-51.

57. Faderl S, Ferrajoli A, Wierda W, et al. Clofarabine combinations as acute myeloid leukemia salvage therapy. Cancer. 2008;113(8):2090-2096.

58. Gidwani P, Ramesh KH, Liu Y, Kolb EA. The combination of clofarabine and cytarabine in pediatric relapsed acute lymphoblastic leukemia: A case report. Chemotherapy. 2008;54(2):120-124.

59. Cooper T, Alonzo T, Gerbing RB, et al. AAML0523: A report from the Children's Oncology Group on the safety of clofarabine in combination with cytarabine in pediatric patients with relapsed acute leukemia. Blood. 2009;114(22): Abstr 3076.

60. Moufarij M, Sampath D, Keating M, Plunkett W. Fludarabine increases oxaliplatin cytotoxicity in normal and chronic lymphocytic leukemia lymphocytes by suppressing interstrand DNA crosslink removal. Blood. 2006;108(13):4187-4193.

61. Bacher U, Klyuchnikov E, Wiedemann B, et al. Safety of conditioning agents for allogeneic haematopoietic transplantation. Expert Opin Drug Saf. 2009;8(3):305-315.

62. Beesley AH, Palmer ML, Ford J, et al. In vitro cytotoxicity of nelarabine, clofarabine and flavopiridol in paediatric acute lymphoblastic leukaemia. Br J Haematol. 2007;137(2):109-116.
Biologics: Targets \& Therapy

\section{Publish your work in this journal}

Biologics: Targets \& Therapy is an international, peer-reviewed journal focusing on the patho-physiological rationale for and clinical application of Biologic agents in the management of autoimmune diseases, cancers or other pathologies where a molecular target can be identified. This journal is indexed on PubMed Central, CAS, EMBase, Scopus

\section{Dovepress}

and the Elsevier Bibliographic databases. The manuscript management system is completely online and includes a very quick and fair peerreview system, which is all easy to use. Visit http://www.dovepress. com/testimonials.php to read real quotes from published authors. 